\title{
Matriz de Bienestar, Escolarización Formal y la Movilidad Social Intergeneracional del Uruguay Contemporáneo
}

\author{
Welfare Matrix, Formal Schooling, and Intergenerational Social \\ Mobility in Contemporary Uruguay
}

\author{
Pablo Menese * \\ Universidad de la República
}

\begin{abstract}
La matriz de bienestar social y la inversión en capital humano dentro del sistema educativo formal obligatorio son elementos estrechamente relacionados. Ambos tienen un rol notable en las posibilidades de movilidad social intergeneracional ascendente de los individuos; no obstante es difícil jerarquizar el rol concreto de cada uno en el proceso de flexibilización -o no- de la estructura social. El artículo se propone analizar la jerarquía del vínculo propuesto en relación a la movilidad social intergeneracional ascendente. Para esto se escogen tres momentos históricos en Uruguay con diferentes regímenes de bienestar, diferentes pautas de movilidad social intergeneracional ascendente, y diferentes niveles educativos formales obligatorios. La estrategia metodológica propone probar distintas hipótesis de mecanismos de asociación causal a través de modelos de tipo loglineal. Los microdatos utilizados son los censos de población de 1963, 1996 y 2011; la población seleccionada son jóvenes varones de entre 18 y 30 años, ocupados, que viven con al menos uno de sus padres también ocupado. Los resultados preliminares sugieren que la inversión en capital humano dentro del sistema educativo formal obligatorio es el mecanismo por el cual ocurre la movilidad social intergeneracional ascendente; con tal, se sugiere que las matrices de bienestar representadas por los periodos censales presentan un vínculo espurio con la movilidad social intergeneracional ascendente.
\end{abstract}

Descriptores: Movilidad social, Educación formal, Bienestar social, Sistema educativo, Educación básica.

The welfare matrix and the investment in human capital are much related inside the compulsory formal schooling. Both have a remarkable role in the odds of ascending intergenerational social mobility. However, it is difficult to hierarchize the role of each in the process of flexibility -or not- of the structure. The article proposes to analyze the hierarchy of the proposed link in relation to the ascending intergenerational social mobility. For this, three historical moments are chosen in Uruguay with different welfare regimes, different patterns of upward intergenerational social mobility, and different compulsory formal education levels. The methodological strategy proposes to test different hypotheses of mechanisms of causal association through loglineal models. The microdata used are the population censuses of 1963, 1996 and 2011; the populations selected are young men between the ages of 18 and 30, employed, living with at least one of their parents also employed. Preliminary results suggest that investment in human capital within the formal compulsory education system is the mechanism by which upward intergenerational social mobility occurs; it is suggested that the welfare matrices represented by the census periods present a spurious link with upward intergenerational social mobility.

Keywords: Social mobility, Formal education, Social welfare, Educational systems, Basic education.

*Contacto: pablo.menese@cut.edu.uy

ISSN: $1696-4713$

www.rinace.net/reice/

revistas.uam.es/reice
Recibido: $\quad 1$ de agosto 2017

$1^{\text {a }}$ Evaluación: 31 de agosto 2017

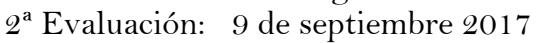

Aceptado: $\quad 20$ de septiembre 2017 


\section{Introducción}

Durante los últimos 50 años han operado una serie importante de cambios que impactan en la movilidad social intergeneracional ascendente. Entre los principales cambios se cuentan la cobertura y servicios de la matriz de bienestar, cambios en los niveles educativos formales obligatorios, y cambios en la estructura productiva, con cambios en las habilidades demandadas por el mercado laboral.

Es plausible pensar que los cambios en los servicios y la cobertura de la matriz de bienestar, la escolarización y la matriz productiva han impactado en las probabilidades de movilidad social intergeneracional ascendente. De todos modos, la forma de asociación de este vínculo y la interacción entre los diversos factores no es clara. El análisis de la asociación y el vínculo de estos factores permite evaluar al menos dos elementos: i) el impacto de la escolarización formal obligatoria en la movilidad social intergeneracional ascendente en diferentes épocas, caracterizadas por diferentes matrices de bienestar; y ii) evaluar el rol del cambio de las matrices de bienestar en la movilidad social intergeneracional ascendente, considerando distintos niveles de escolarización formal obligatoria.

La relevancia social del artículo radica en que permite evaluar el peso relativo entre la escolarización formal obligatoria y las matrices productiva y social en las oportunidades de movilidad social; ergo, la contribución a la equidad dentro de la estructura social que diferentes estados bienestar manifiesta en uno de sus agentes con la pretensión del alcance universal.

La relevancia científica del artículo trata de realizar al menos dos aportes. En primer lugar, armoniza los patrones de movilidad social intergeneracional los últimos 50 años, generando un insumo que permite evaluar al menos dos elementos: i) avances y retrocesos del mercado de empleo y ii) el impacto de la matriz productiva en la estructura social. En segundo lugar, un análisis del peso de la escolarización -ceteris paribus la movilidad social intergeneracional ascendente- aporta en el debate de las transiciones a la adultez, más particularmente en la relación entre las trayectorias educativas y laborales.

\section{Marco teórico y antecedentes}

La movilidad social puede entenderse como la relación que existe en una posición de la estructura social en un $t_{0}$, con respecto a la posición en la estructura social en un $t_{1}$ (Boado, 2008). Este desplazamiento puede ocurrir para un mismo individuo, para una generación con respecto a otra (Boado, 2004; Goldthorpe, 1987). Esta posición en la estructura social puede ser operacionalizada de distintas formas, utilizando distintas escalas o esquemas, con distinto poder predictivo y marco explicativo. Se puede utilizar un esquema basado principalmente en la actividad laboral como el esquema neo-marxista (Wright, 1997), o escalas basadas en el prestigio ocupacional (Ganzeboom, 1996). A efectos del presente trabajo se utiliza el esquema neo-weberiano, para el cual el prestigio ocupacional depende de dos atributos: i) las credenciales educativas necesarias para el ejercicio de la tarea asociada y ii) la capacidad de supervisión de la tarea asociada (Goldthorpe y Erikson, 1993). 
Este último tipo de esquema ha sido utilizado a nivel nacional tanto para la estimación del efecto del hogar de origen en las desigualdades educativas, como también para esquemas de movilidad social intergeneracional (Fernández y Boado, 2010; Menese, 2014; Ríos, 2014). En su versión resumida a tres categorías genera tres clases sociales ordenadas desde Servicio (gerentes, directivos y profesionales), Intermedia (empleados no manuales) y Trabajadora (trabajadores manuales calificados y no calificados). En este caso la movilidad social intergeneracional está pautada en el cuadro 1.

Cuadro 1. Esquema de movilidad social

\begin{tabular}{lccc}
\hline & \multicolumn{3}{c}{ EMPLEO EN t$_{1}$} \\
\cline { 2 - 4 } & Servicio & Intermedia & Trabajadora \\
\hline Servicio & 0 & - & - \\
Intermedio & + & 0 & - \\
Trabajadora & + & + & 0 \\
\hline
\end{tabular}

Fuente: Elaboración propia.

En el cuadro 1 se observa la diagonal principal marcada con "o" lo cual implica reproducción de la posición en la estructura social entre $t_{0} \mathrm{y} \mathrm{t}_{1}$. El cuadrante inferior izquierdo "+" representa movilidad social ascendente entre $t_{0} y t_{1}$. El cuadrante superior derecho "-" implica movilidad social descendente entre $t_{0} \mathrm{y}_{1}$.

En cualquier sociedad la movilidad social intergeneracional habla de la rigidez o flexibilidad de la estructura social entre distintas cohortes generacionales. De este modo sociedades con poca movilidad social intergeneracional, implican patrones reproductivos en los tipos de empleo entre las distintas cohortes. Por otro lado, sociedades con alta movilidad social intergeneracional presentan panoramas de mayor equidad donde el hogar de origen de los individuos es menos determinante de su destino ocupacional (Boado, 2008; UNESCO, 2016). Un tipo de movilidad social intergeneracional de interés es la de tipo ascendente, donde los individuos logran ocupar un mejor sitio que sus padres en la estructura social. Este tipo específico de movilidad social -superando el hogar de origen- sugiere estímulos particulares administrados a una nueva generación por parte del hogar de origen, agentes de bienestar, o impulsos del propio individuo.

Estos estímulos actúan con diversos mecanismos que incrementan o disminuyen las posibilidades de movilidad social intergeneracional ascendente; algunos de estos mecanismos operan de manera particularista y con aprovechamientos limitados, tal es el caso del capital social, el cual únicamente opera en quienes pertenecen o refrieren a determinados tipos de redes con determinadas características y clausuras (Lin, 2001). Un segundo tipo de mecanismo de mayor alcance que los particularistas son las políticas sociales, el cual se puede asociar al estado de bienestar y los agentes de bienestar (Esping-Andersen, 1990) cuya cobertura varía de acuerdo al alcance de la matriz de bienestar desde regímenes universales, hasta regímenes corporativos (Busquets, 2009; Midaglia, 2009).

Uno de los agentes de bienestar con pretensión universalista en las matrices de bienestar es el sistema educativo formal obligatorio (Ríos, 2014). Son varios los objetivos y las externalidades de la obtención de credenciales educativas en las sociedades modernas, en general intentan democratizar determinado tipo de conocimiento entre los habitantes de un territorio, más allá de cualquier otra condición que residir en dicho territorio. La forma de uniformizar los contenidos necesarios a ser democratizados, se determinan a 
través de los programas académicos nacionales habilitados por el gobierno de la educación, donde se desarrollan contenidos que una sociedad considera mínimo necesario para su desarrollo y reproducción (Da Silveira, 2009).

A través del sistema educativo, como agente de bienestar universal, los individuos acceden a competencias e invierten en capital humano, el cual será reconocido -o no- de acuerdo a las necesidades del mercado de trabajo y de este modo lograr distintas trayectorias de movilidad social intergeneracional. Ahora bien, lo que una sociedad considera mínimo necesario para su desarrollo y reproducción ha variado a lo largo del último siglo. Esto responde a varios elementos, entre ellos cambios en la matriz productiva y la consiguiente necesidad de ciudadanos con mayores competencias y tecnificación. En el caso de Uruguay a finales del siglo XIX se establece como nivel educativo formal obligatorio Primaria, el cual persiste hasta 1971 cuando se extiende hasta Educación Media Básica, y posteriormente en 2008 hasta Educación Media Superior (Menese y Ríos, 2013).

De acuerdo a lo expuesto hasta ahora parece evidente la importancia del modelo de estado bienestar en términos de alcance y servicios. Al respecto los años escogidos para el análisis representan tres tipos distintos de matrices de bienestar en Uruguay. Durante 1963 una matriz de protección social universal de tipo corporativa, con acceso a los servicios de bienestar de acuerdo a la afiliación a ciertas corporaciones, con escasos servicios focalizados orientados a los sectores más vulnerables, en particular se destaca la usencia de programas de persistencia y reinclusión dentro del sistema educativo. En 1996 operaba un escenario que había sido reformado en función de los procesos de liberalización económica, donde se comienza a desfinanciar el sistema de seguridad social y los ciclos de la pobreza son más sensibles a los ciclos económicos. Por ultimo 2011 se caracteriza por un sistema hibrido con programas universales y focalizados muchos de ellos orientados a la reinclusón y persistencia educativa (Mancebo y Goyeneche, 2010; Midaglia, 2009)

La democratización de la enseñanza formal y el incremento de las credenciales educativas obligatorias, se acompasan con distintos mecanismos de amortiguación de las desigualdades en virtud de diferentes modelos de matriz de bienestar; la asociación e interacción de estos elementos genera distintas chances de movilidad social intergeneracional ascendente, o no. Ergo, parece pertinente cuestionar si la extensión de años de educación desde mediados del siglo Xx y comienzos del siglo XXI acompaso verdaderamente el cambio en la matriz productiva y la necesidad de competencias en un proceso de división social del trabajo marcado por el desarrollo tecnológico, o si por otro lado fueron los distintos servicios y coberturas de la seguridad social quienes han amortiguado las desigualdades del hogar de origen.

Por último, esta discusión no se agota en la escolarización, la movilidad social intergeneracional y la matriz productiva. Existe un debate sobre la escolarización y el valor de la modalidad de enseñanza y de las credenciales educativas en el mercado laboral, particularmente centrado en la transición entre la escuela y el trabajo (Gangl, Müller y Raffe, 2003). Por un lado, existe una vocación por educar de espaldas al mercado laboral, como medio de liberación y lucha contra la inequidad del sistema capitalista (Freire, 1987), y una variante de esto es educar en artes liberales como propedéutico pre-universitario (Kang y Bishop, 1989), mientras que la postura opuesta es la de contribuir en la escolarización como vía de inversión en capital humano (Becker, 
1964). Uruguay no está exento de este debate (Filgueira et al., 2014), por lo cual aportar evidencia en torno a algún sentido de la discusión tiene ecos nacionales e internacionales en el vínculo educación trabajo.

\section{Objetivos e hipótesis}

El objetivo del artículo es despejar el factor preponderante en la movilidad social intergeneracional ascendente al evaluar la relación de la misma con la escolarización formal obligatoria, y los cambios de la matriz de bienestar social los últimos 50 años.

De acuerdo a la metodología de análisis propuesta se podría ensayar un total de nueve hipótesis, algunas de ellas sin sentido teórico ninguno; con lo cual se opta contratar un conjunto de hipótesis teóricamente relevantes asociados al objetivo planteado.

Hipótesis 1: Independencia mutua [movilidad] [estudio] [año]

No existe relación entre la movilidad social intergeneracional ascendente, la acreditación de la escolarización formal obligatoria, y el periodo censal.

Hipótesis 2: Independencia parcial [movilidad estudio] [año]

La movilidad social intergeneracional ascendente se asocia a la obtención de la acreditación formal obligatoria, esta asociación opera ceteris paribus para con los atributos de la matriz de bienestar anidados en las características del periodo censal.

\section{Hipótesis 3: Independencia parcial [movilidad año] [estudio]}

La movilidad social intergeneracional ascendente se asocia con los atributos de la matriz de bienestar anidados en las características del periodo censal, esta asociación opera es ceteris paribus a la obtención de la acreditación formal obligatoria.

\section{Hipótesis 4: Independencia condicional [movilidad año] [estudio año]}

Existe una asociación espuria entre la movilidad social intergeneracional ascendente y haber acreditado la escolarización formal obligatoria. Con lo cual la movilidad social intergeneracional ascendente y la acreditación de la escolarización formal obligatoria se asocian cada una los atributos de la matriz de bienestar anidados en las características del periodo censal.

\section{Hipótesis 5: Asociación homogénea [movilidad estudio] [movilidad año] [año estudio]}

Existen asociaciones parciales entre los distintos factores, que son constantes con otras asociaciones parciales de factores. La movilidad social intergeneracional ascendente se asocia con haber obtenido la acreditación de la escolarización formal obligatoria, esto es constante a través de una relación entre la movilidad social intergeneracional ascendente y los atributos de la matriz de bienestar anidados en las características del periodo censal; todo esto ocurre con distintas pautas de acreditación de la educación formal obligatoria en cada periodo censal.

\section{Hipótesis 6: Modelo saturado [movilidad estudio año]}

Este modelo propone que todos los factores están asociados entre sí. Este modelo es teóricamente débil en virtud de que no aclara ni sugiere los mecanismos por los cuales ocurren las distintas asociaciones. 


\section{Diseño, método, técnica y datos}

Diseño, método y técnica

El diseño de investigación es de tipo descriptivo y explicativo. Se propone un análisis de tipo cuantitativo utilizando datos secundarios. Para el plan de análisis se propone contrastar las hipótesis planteadas utilizando como técnica los modelos de tipo loglineal, los cuales plantean un modelo teórico de relaciones entre factores a ser empíricamente contrastados en virtud de las distribuciones de los individuos en los diferentes espacios lógicos. Este método de comparación entre frecuencias observadas, y distintas recetas de frecuencias esperadas permite adicionalmente contrastar los distintos espacios de desajuste al utilizar residuos ajustados, con lo cual, cada hipótesis es contrastada tanto en el mecanismo propuesto, como en elementos parciales que hace al mismo (Agresti, 1990).

Retomando las hipótesis ya planteadas, se propone ir desde el llamado modelo vacío, hacia el modelo saturado pasando por distintas propuestas de asociación adjuntas a diferentes hipótesis sobre el mecanismo entre movilidad social intergeneracional ascendente, escolarización formal obligatoria, y el periodo de estudio, es decir, una estrategia tipo forward (Boado, 2013).

La predicción concreta de cada celda, en un escenario de tres variables Formalmente el modelo saturado se expresa como:

$$
\log n_{i j k}=\lambda+\lambda_{i}^{x}+\lambda_{j}^{y}+\lambda_{k}^{z}+\lambda_{i j}^{x y}+\lambda_{i j}^{x z}+\lambda_{j k}^{y k}+\lambda_{i j k}^{x y z}
$$

En virtud de los microdatos se cuenta con una gran cantidad de casos, el método loglineal es sensible a análisis con gran cantidad de casos en las distintas distribuciones, con lo cual se realiza una simple operación de división de los casos en la celda. De este modo, manteniendo la proporción de casos por celda, se cuenta con un volumen en el conteo de casos que permiten el análisis sin grandes alteraciones en los residuos, ergo en los resultados más generales de los distintos modelos de análisis.

\section{Datos}

Los datos que se utilizan son de tipo secundarios y se corresponden a los microdatos de los censos nacionales de hogares y población de 1963, 1996 y 2011. Estos datos han sido escogidos por dos razones: i) cada año tiene un nivel educativo formal obligatorio: Primaria en 1963, Educación Media Básica en 1996, y Educación Media Superior en 2011; y ii) si bien los periodos entre censos son dispares la comparación 1963-1996 se asemeja a la comparación 1959-1996 realizada por Boado utilizando los trabajos de Labbens y Solari (Boado, 2004), con lo cual permite un dialogo con antecedentes recientes.

La sub-muestra que se escoge para el análisis corresponde a: i) varones ii) los jóvenes de entre 18 y 30 años ocupados; iii) que viven con al menos uno de sus padres; y iv) al menos uno de dichos padres también está ocupado. El cuadro 3 (del apartado 4.1.) explicita la distribución del total de la población para cada periodo. La razón de escoger únicamente varones radica en al menos dos razones, la primera es que en general -a cualquier edad- la participación de las mujeres en el mercado laboral es menor que la de los hombres. En segundo lugar, a lo largo de los últimos 50 años la participación de la mujer en mercado laboral se ha ido incrementando. Teniendo en cuenta estas tendencias, 
incluir las mujeres en el análisis generaría un efecto no aleatorio asociado a la participación femenina en el mercado laboral, y sus oportunidades dentro del mismo que necesariamente generaría una sobrestimación/subestimación de los distintos coeficientes.

Entre las ventajas de utilizar estos microdatos radica en que más allá de sus problemas de constructo constituyen el mejor tipo de datos en Uruguay. Adicionalmente considerando los requisitos de la técnica de análisis, contar con microdatos que incluyen totales poblacionales permite limitar al máximo la acumulación de celdas vacías. Las desventajas de utilizar estos microdatos son al menos tres. En primer lugar, el censo de 1963 no fue validado por el Instituto Nacional de Estadística, el responsable de este proceso fue el Centro Latinoamericano y Caribeño de Demografía (CELADE) y han sido obtenidos por el autor a través del Banco de Datos de la Facultad de Ciencias Sociales. Los datos fueron recopilados a partir de datos secundarios parciales en formato cintas magnéticas, y corroborado con los informes oficiales de la época que se encontraron disponibles.

En segundo lugar y también asociado al constructo de los microdatos, el censo del 2011 presentó problemas durante su codificación en la identificación de la categoría ocupacional de los encuestados, con lo cual las mismas fueron codificadas en función del código Clasificador Internacional Uniforme de Ocupaciones (CIUO-08 del inglés ISCO-08) a dos dígitos, es decir grandes grupos ocupacionales y subgrupos principales. Ergo, la codificación de clase social para este último censo es menos confiable. Por su parte el censo 1963 se codifico con el código COTA-70, mientras que el censo 1996 esta codificado a CNUO-95. Estas múltiples codificaciones requirieron de trabajo de conversión con el fin de la armonización y construcción de las distintas clases sociales.

Por último, los datos de los censos no están hechos para detectar la movilidad social intergeneracional en virtud de que no pregunta el empleo de los padres de los encuestados, con lo que la movilidad social intergeneracional únicamente se puede recoger dentro de los hogares con padres e hijos ocupados. Por este motivo es que se realizó la muestra cómo se especifica en el apartado correspondiente: jóvenes entre 18 y 30 años activos que viven con padres activos. Existen al menos dos limitaciones en este enfoque al respecto del presente artículo: i) la población de los 18 a los 30 años se desgrana de acuerdo a la emancipación del hogar de origen, la cual es mayor hacia el final de la edad señalada. De este modo no se encuentran en la estimación de aquellos que se han emancipados, este retraso está asociado al nivel socioeconómico del hogar de origen (Ciganda y Pardo, 2014), mientras que la edad de salida del hogar de origen no ha sido estable durante los últimos 50 años, más recientemente los calendarios de emancipación se han retrasado (Ciganda y Pardo, 2014), máxime considerando la extensión de años de educación formal obligatoria (Filardo, 2010). El cuadro 2 resume las variables utilizadas en el análisis, así como el concepto al que corresponde y el método de codificación.

\section{Análisis}

Se propone un análisis en dos momentos, en primer lugar descriptivo de las distribuciones, y en segundo lugar del modelo loglineal. 
Cuadro 2. Operacionalización de los factores propuestos para el análisis

\begin{tabular}{|c|c|c|}
\hline VARIABLE & CONCEPTO & Código \\
\hline $\begin{array}{l}\text { Movilidad social } \\
\text { intergeneracional } \\
\text { ascendente }\end{array}$ & $\begin{array}{l}\text { Utilizando el esquema de clase social EGP } \\
\text { codificada a } 3 \text { categorías (Erikson, } \\
\text { Goldthorpe y Portocarero, 1979) se } \\
\text { identifica a aquellos individuos con } \\
\text { movilidad social intergeneracional } \\
\text { ascendente. }\end{array}$ & $\begin{array}{l}\text { Toma valor } 1 \text { para aquellos } \\
\text { jóvenes con movilidad social } \\
\text { intergeneracional } \\
\text { ascendente. o para todos los } \\
\text { demás. }\end{array}$ \\
\hline $\begin{array}{l}\text { Estudio formal } \\
\text { obligatorio }\end{array}$ & $\begin{array}{l}\text { Identifica a quienes han obtenido la } \\
\text { acreditación del nivel educativo formal } \\
\text { obligatorio. } \\
\text { La educación formal obligatoria en } 1963 \\
\text { era Primaria completa, en } 1996 \\
\text { Educación Media Básica completa, y en } \\
\text { 2011 Educación Superior completa. }\end{array}$ & $\begin{array}{l}\text { Toma valor } 1 \text { para aquellos } \\
\text { jóvenes que han obtenido la } \\
\text { acreditación del nivel formal } \\
\text { obligatorio. o para los demás }\end{array}$ \\
\hline Año censal & $\begin{array}{l}\text { Identifica el año en el que se realizó el } \\
\text { censo al cual pertenecen los distintos } \\
\text { jóvenes. } \\
\text { Cada uno de estos años implica al menos } \\
\text { dos cosas: i) una matriz productiva } \\
\text { diferente con todas las dinámicas en el } \\
\text { mercado de trabajo que implica; y ii) una } \\
\text { matriz de bienestar con distintas } \\
\text { coberturas y servicios. }\end{array}$ & $\begin{array}{l}\text { Toma valor } 1 \text { para el año } \\
\text { censan } 1963,2 \text { para el año } \\
\text { censal 1996, y } 3 \text { para el año } \\
\text { censal } 2011 .\end{array}$ \\
\hline
\end{tabular}

Fuente: Elaboración propia.

\subsection{Análisis descriptivo}

El primer análisis corresponde a la descripción de la distribución de individuos en los distintos espacios lógicos para cada periodo (cuadro 3). Los ejes que caracterizan cada espacio son: i) haber logrado movilidad social ascendente contra no haberla logrado; ii) haber acreditado la educación formal obligatoria contra no haberla acreditado; y iii) el periodo caracterizado por el año del censo.

Cuadro 3. Distribución de jóvenes según movilidad social intergeneracional, nivel educativo formal obligatorio y periodo censal

\begin{tabular}{lcccccc}
\hline & \multicolumn{2}{c}{1963} & \multicolumn{2}{c}{1996} & \multicolumn{2}{c}{ 2011 } \\
\cline { 2 - 7 } & $\begin{array}{c}\text { Sin nivel } \\
\text { obligat. }\end{array}$ & $\begin{array}{c}\text { Nivel } \\
\text { obligat. }\end{array}$ & $\begin{array}{c}\text { Sin nivel } \\
\text { obligat. }\end{array}$ & $\begin{array}{c}\text { Nivel } \\
\text { obligat. }\end{array}$ & $\begin{array}{c}\text { Sin nivel } \\
\text { obligat. }\end{array}$ & $\begin{array}{c}\text { Nivel } \\
\text { obligat. }\end{array}$ \\
\hline Sin movilidad social & 15.306 & 8.494 & 47.045 & 17.035 & 47.940 & 12.101 \\
ascendente & $(78 \%)$ & $(62 \%)$ & $(86 \%)$ & $(73 \%)$ & $(88 \%)$ & $(67 \%)$ \\
\hline Movilidad & 4.313 & 5.183 & 7.973 & 6.333 & 6.371 & 5.900 \\
ascendente & $(22 \%)$ & $(38 \%)$ & $(14 \%)$ & $(27 \%)$ & $(12 \%)$ & $(33 \%)$ \\
\hline \multirow{2}{*}{ Total parcial } & 19.619 & 13.677 & 55.018 & 23.368 & 54.311 & 18.001 \\
& $(100 \%)$ & $(100 \%)$ & $(100 \%)$ & $(100 \%)$ & $(100 \%)$ & $(100 \%)$ \\
\hline Total absoluto & \multicolumn{2}{c}{33.296} & 78.386 & \multicolumn{2}{c}{72.312} \\
\hline
\end{tabular}

Fuente: Elaboración propia a partir de los datos del Censo 1963, 1996 y 2011.

El cuadro 3 muestra para cada año la frecuencia y el porcentaje de quienes para cada año censal habían acreditado y no la educación formal obligatoria junto con quienes habían logrado y no movilidad social intergeneracional ascendente. Al comenzar el análisis por periodo del censo, se observa en general que el año donde se observa mayor porcentaje 
de movilidad social intergeneracional ascendente es 1963, con un 28\%, seguido por 1996 con un $18 \%$ y por ultimo 2011 con un $17 \%$. Esto ocurre asociado a otros dos elementos: i) 1963 es el año con mayor porcentaje de personas con el nivel formal obligatorio acreditado con un 41\%, mientras que en 1996 lo había hecho un 30\% y en 2011 un 25\% y ii) los niveles de escolarización formal obligatorios, y el supuesto de capacidades y habilidades obtenidas para cada año es sensiblemente menor siendo Primaria en 1963, Educación Media Básica en 1996, y Educación Media Superior en 2011. Esto sugiere una de dos cosas, o bien la matriz de bienestar de 1963 amortiguaba con mayor eficiencia las desigualdades sociales de origen que en 1996 y 2011, así como 1996 también es más eficiente que 2011; o bien las competencias que brindaba el sistema educativo formal obligatorio en 1963 eran una inversión en capital humano más eficiente y mejor reconocida con respecto a las competencias brindadas en 1996 y 2011, así como 1996 con respecto a 2011 .

Al observar lo que ocurre a la interna de cada año, entre quienes alcanzaron el nivel educativo formal obligatorio, encontramos nuevamente que 1963 -con un 38\%-es el año en el cual mayor cantidad de individuos logran movilidad social intergeneracional ascendente una vez acreditado el nivel educativo formal obligatorio, seguido por 2011 con un 33\%, y por último 1996 con un 27\%. La diferencia entre los guarismos en este caso es mucho menos que al observar en el conjunto de los años. Esto permite pensar que la diferencia en la movilidad social intergeneracional ascendente en los distintos periodos se asocia con los atributos de dichos periodos, así como la escolarización se asocia también a los atributos del periodo siendo espuria la relación entre movilidad social intergeneracional ascendente y la escolarización formal obligatoria.

\subsection{Análisis loglineal}

En el apartado anterior se estudiaron las distintas distribuciones sin mayores pretensiones que las de un análisis descriptivo. No obstante, dichas distribuciones sugieren contrastes empíricos que hacen eco en las hipótesis planteadas en el vínculo movilidad social intergeneracional ascendente, acreditación de la escolarización formal obligatoria, y atributos del periodo censal. Con tal a continuación se propone el análisis de tipo loglineal, para realizar contrastes empíricos más exigentes de los tipos de asociación planteados.

El cuadro 4 resume el ajuste para cada una de las hipótesis planteadas, donde únicamente se rechaza el ajuste propuesto por la hipótesis de independencia mutua, con lo cual, el modelo que postula que la movilidad social ascendente, la acreditación de la escolarización formal obligatoria y el periodo censal no se relacionan no es empíricamente contrastable.

Cuadro 4. Ajuste de las hipótesis propuestas en los modelos loglineal

\begin{tabular}{|c|c|c|c|c|c|c|c|}
\hline HIPÓTESIS & MODELO & $\mathrm{G}^{2}$ & DF & $\mathbf{P}$ & RESULTADO & DISIMILARIDAD & BIC \\
\hline Indep. mutua & {$[\mathrm{u}][\mathrm{s}][\mathrm{y}]$} & 22,362 & 7 & 0,002 & rechazo & 9,3 & 4,968 \\
\hline Indep. parcial & {$[\mathrm{u} s][\mathrm{y}]$} & 8,778 & 6 & 0,801 & acepta & 5,6 & $-6,132$ \\
\hline Indep. parcial & {$\left[\begin{array}{ll}\mathrm{u} & \mathrm{y}\end{array}\right][\mathrm{s}]$} & 18,468 & 5 & 0,244 & acepta & 8,7 & 6,043 \\
\hline $\begin{array}{l}\text { Indep. } \\
\text { condicional }\end{array}$ & {$\left[\begin{array}{ll}\mathrm{u} & \mathrm{y}\end{array}\right]\left[\begin{array}{ll}\mathrm{s} & \mathrm{y}\end{array}\right]$} & 12,932 & 3 & 0,907 & acepta & 6,7 & 5,477 \\
\hline $\begin{array}{l}\text { Interacción } \\
\text { homogénea }\end{array}$ & $\begin{array}{c}{\left[\begin{array}{ll}\mathrm{u} & \mathrm{s}\end{array}\right]\left[\begin{array}{ll}\mathrm{u} & \mathrm{y}\end{array}\right]} \\
{\left[\begin{array}{ll}\mathrm{y} & \mathrm{s}\end{array}\right]}\end{array}$ & 0,820 & 2 & 1,000 & acepta & 1,7 & $-4,150$ \\
\hline
\end{tabular}

Fuente: Elaboración propia a partir de los datos del Censo 1963, 1996 y 2011. 
El resto de los modelos cumplen todos la condición de aceptar las diferentes hipótesis propuestas, aunque con distintos ajustes. Esto ocurre en virtud a que tanto el vínculo teórico como el vínculo empírico de los tres factores mencionados son altamente plausibles para cada hipótesis.

Aun así, hay dos modelos en particular que resaltan, en primer lugar, el de independencia parcial que postula la relación $[\mathrm{u} \mathrm{s}][\mathrm{y}]$, y en segundo lugar el modelo de interacción homogénea. En el caso del modelo de interacción homogénea es razonable siendo que es el modelo más cercano al modelo saturado, el cual postula múltiples asociaciones entre los factores, de todos modos tiene muchos grados de libertad. Por tal el modelo que se escoge es el de independencia parcial por al menos cuatro motivos: i) gran cantidad de grados de libertad, es decir es un buen modelo explicativo consumiendo pocos grado de libertad; ii) de acuerdo al índice de disimilaridad se lograría un ajuste perfecto con la redistribución del 5.6\% de los casos; iii) el criterio de información bayesiana (BIC) es el menor del total de los modelos, con lo cual es el de mayor parsimonia y poder explicativo; y iv) es el modelo que teóricamente logra una explicación más plausible frente al modelo de interacción homogénea.

El cuadro 5 muestra los residuos ajustados para cada modelo, valores por encima de 1,96 y por debajo de $-1,96$ implican celdas con distribuciones que desajustan significativamente con la hipótesis propuesta con el modelo respectivo. Al observar el modelo de interacción parcial escogido observamos que todas las celdas presentan valores no significativos, con lo cual no hay grandes distribuciones empíricas que desajusten con las distribuciones teóricas propuestas.

Cuadro 5. Residuos ajustados de los modelos loglineal

\begin{tabular}{|c|c|c|c|c|c|c|c|}
\hline MOVILIDAD & ESTUDIO & AÑo & {$[\mathbf{u}][\mathbf{s}][\mathbf{y}]$} & {$\left[\begin{array}{ll}\mathbf{u} & \mathbf{s}\end{array}\right][\mathrm{y}]$} & {$\left[\begin{array}{ll}\mathbf{u} & \mathbf{y}\end{array}\right][\mathbf{s}]$} & {$\left[\begin{array}{ll}\mathbf{u} & \mathbf{y}\end{array}\right]\left[\begin{array}{ll}\mathbf{s} & \mathbf{y}\end{array}\right]$} & $\begin{array}{c}{\left[\begin{array}{cc}\mathbf{u} & \mathbf{s}\end{array}\right]\left[\begin{array}{ll}\mathbf{u} & \mathbf{y}\end{array}\right]} \\
{\left[\begin{array}{ll}\mathbf{y} & \mathbf{s}\end{array}\right]}\end{array}$ \\
\hline No & No acredita & 1963 & $-1,13$ & $-1,47$ & $-0,48$ & 0,48 & $-0,11$ \\
\hline $\mathrm{Si}$ & No acredita & 1963 & $-0,17$ & 0,72 & $-1,28$ & $-0,77$ & 0,22 \\
\hline No & Acredita & 1963 & 0,24 & 0,91 & 0,73 & $-0,58$ & $\mathrm{O}, 15$ \\
\hline $\mathrm{Si}$ & Acredita & 1963 & 3,27 & 1,62 & 1,97 & 0,92 & $-0,18$ \\
\hline No & No acredita & 1996 & 0,61 & 0,01 & 0,45 & 0,44 & $-0,10$ \\
\hline $\mathrm{Si}$ & No acredita & 1996 & $-1,21$ & 0,01 & $-0,92$ & $-0,92$ & 0,25 \\
\hline No & Acredita & 1996 & $-0,59$ & 0,35 & $-0,69$ & $-0,67$ & 0,17 \\
\hline $\mathrm{Si}$ & Acredita & 1996 & 1,14 & $-0,56$ & 1,40 & 1,42 & $-0,26$ \\
\hline No & No acredita & 2011 & 1,59 & 0,99 & 1,28 & 0,60 & 0,16 \\
\hline $\mathrm{Si}$ & No acredita & 2011 & $-1,60$ & $-0,50$ & $-1,07$ & $-1,33$ & $-0,42$ \\
\hline No & Acredita & 2011 & $-1,79$ & $-0,99$ & $-1,96$ & $-1,04$ & $-0,31$ \\
\hline $\mathrm{Si}$ & Acredita & 2011 & 1,14 & $-0,51$ & 1,65 & 2,30 & 0,49 \\
\hline
\end{tabular}

Fuente: Elaboración propia a partir de los datos del Censo 1963, 1996 y 2011.

El otro modelo de interacción parcial propuesto tiene desajuste en quienes logran movilidad social intergeneracional ascendente, habiendo acreditado educación formal obligatoria, en 1963; y entre quienes no logran movilidad social intergeneracional ascendente, habiendo acreditado educación formal obligatoria, en 2011. En el primer caso hay demasiados casos en las frecuencias observadas en relación a las esperadas, en el segundo caso faltan casos en las frecuencias observadas en relaciona a las esperadas. El modelo de interacción condicional muestra mayor cantidad de casos observados en relación a los esperados en la celda de quienes logran movilidad social intergeneracional 
ascendente, habiendo acreditado educación formal obligatoria, en 2011. Una alternativa frente a estos desajustes pudiera ser incluir una variable tipo muda que clausure las celdas cuyos residuos desajustan y reestimar el modelo en particular. Esto no se aplica en virtud de que el modelo de independencia parcial escogido - [ $\mathrm{u} s][\mathrm{y}]-$ satisface las pretensiones explicativas.

En virtud del problema de investigación los modelos de independencia parcial eran particularmente atractivos, dichos modelos implican que o bien el sistema educativo amortigua las desigualdades del hogar de origen que la matriz de bienestar no puede; o bien que la matriz de bienestar amortigua la desigualdad del hogar de origen que el sistema educativo no puede. La hipótesis que encontró un mejor contraste empírico es la primera, en la cual parece que sistema educativo amortigua las desigualdades del hogar de origen que la matriz de bienestar no puede. Es decir, en distintos periodos, con distintas matrices de bienestar y con distintas matrices productivas la acreditación de la educación formal obligatoria incrementa las chances de romper con la herencia ocupación del hogar de origen y evitar la movilidad social intergeneracional descendente. Este hallazgo es particularmente interesante cuando se considera las distintas competencias y habilidades que necesariamente brindaban niveles educativos formales obligatorios diferentes. Esto sugiere que el sistema educativo ha logrado deflactar en cada periodo las competencias y habilidades necesarias de acuerdo a las demandas del mercado laboral, y al alcance y servicios de la matriz de protección social.

\section{Conclusiones}

En primer se debe recordar las limitaciones de la muestra para precisar el alcance de las inferencias. El poder de generalización se restringe - para cada año censal- a: i) varones: ii) entre 18 y 30 años ocupados; iii) que viven con al menos uno de sus padres; y iv) al menos uno de dichos padres también está ocupado. A pesar de estas limitaciones, se reafirmar la elección de los microdatos, ya que no existen en Uruguay datos de similares características para construir tal tipo de análisis. Con lo cual -incluso imperfectas- las conclusiones permiten una primera aproximación al fenómeno.

El hallazgo sustantivo sugiere que la asociación entre movilidad social intergeneracional ascendente y acreditar la escolarización formal obligatoria es independiente del periodo censal. Parece evidente entonces que en todo periodo alcanzar la escolarización formal obligatoria incrementa las chances de romper con la dinámica origen/destino, esto ocurre incluso en modelos de estado bienestar con matrices productivas bien distintas. Esto implica que aquellos que acreditaron la educación formal obligatoria en 1963 no presenten diferencias con quienes lo hicieron en 1996 y 2011. Esto sugiere que 3 y 6 años más de escolarización, y el ingreso a un nuevo nivel educativo (Educación Media), tenían el mismo reconocimiento y oportunidades de movilidad social intergeneracional ascendente en todas las matrices de bienestar y productivas asociadas a los diferentes periodos. Esto podría sugerir que el incremento de la obligatoriedad establecida en 1971 y 2008 logro deflactar las habilidades y conocimientos valorados como necesarios por el mercado laboral. Por lo tanto, la escolarización parece haber amortiguado las desigualdades origen/destino flexibilizando la estructura social en los tres periodos, sin diferencias significativas entre 1963, 1996 y 2011. 
Considerando los resultados globales, se puede pensar que las desigualdades origen/destino o bien pueden ser influidas por una matriz de bienestar y mercado laboral que amortigüe las desigualdades, o bien por un sistema educativo que democratice las oportunidades; en el Uruguay de los últimos 50 años pareciera ser el caso de un sistema educativo que democratiza las oportunidades y flexibiliza la estructura social. Resta pensar en clave prospectiva en un escenario en el que los niveles educativos formales obligatorios no alcanzan una cobertura universal. En estos casos, más allá de la capacidad del sistema educativo de amortiguar las desigualdades del hogar de origen deflactándose a sí mismo, queda muy restringido a quienes alcanzan los niveles establecidos como formales obligatorios. Es aquí donde la matriz de bienestar debiera actuar para re-incluir jóvenes que se han desafiliado, o contribuir a la persistencia de los mismos, mientras genera nuevos servicios que contribuyan la disminución de las desigualdades en función del hogar que toco nacer.

Por último, la evidencia en Uruguay apunta en dos sentidos trascendentes en relación al estado de la discusión sobre las credenciales educativas y su impacto en el mercado laboral. En primer lugar, al mercado laboral le importa lo que ocurre dentro del sistema educativo formal obligatorio, y lo reconoce por encima de consideraciones estructurales. Por lo tanto, bien podemos continuar educando de espaldas al mercado laboral, o reconocer que una complementariedad entre la esfera educativa y laboral redunda en mayor bienestar para los estudiantes. En segundo lugar, países como Uruguay que presentan fuertes desigualdades educativas en el acceso y la acreditación, debieran tener mecanismos amortiguadores dentro de la matriz de bienestar que contribuya a flexibilizar la estructura social. De acuerdo a los datos presentados, durante los últimos 50 años en Uruguay no ha habido agentes de bienestar que intervengan en la ecuación hogar de origen-escolarización-destino ocupacional. De continuar esta tendencia, el beneficio de la acreditación de la escolarización formal queda en manos de unos pocos que logran la credencial, y en general, estos pocos pertenecen a las clases más acomodadas, incrementando el espiral de la desigualdad a escala intergeneracional.

Por último, para futuros trabajos se propone ampliar la varianza en dos sentidos: i) generar comparaciones entre países, con el mismo nivel de escolarización formal obligatoria ampliando los modelos de matriz de bienestar propuestos, con el fin de observar si persiste el esquema de independencia parcial [movilidad-escolarización] [matriz de bienestar]; y ii) ampliar los modelos de los sistema educativos formales, manteniendo constante los años de escolarización formal y las matrices de bienestar, con el fin de observar si persiste el esquema de independencia parcial [movilidadescolarización] [matriz de bienestar]. De persistir el esquema bajo las dos pruebas, habría que cuestionar la estructura de la herencia y de la reproducción de la estructura social, como principal fuente de desigualdad, tanto para la escolarización, como para la movilidad social ascendente.

\section{Referencias}

Agresti, A. (1990). Categorical data analysis. Nueva York, NY: John Wiley.

Becker, G. S. (1964). Human capital. The Journal of Human Resources, 2(1), 97-104. https://doi.org/10.2307/144593 
Boado, M. (2004). Herencia y movilidad social en Montevideo 1959-1996: Tras los pasos de Labbens y Solar. En E. Mazzei (Ed.), Uruguay desde la sociología (pp. 195-226). Montevideo: Universidad de la Republica.

Boado, M. (2008). La movilidad social en el Uruguay contemporaneo. Montevideo: UDELARIUPERJ.

Boado, M. (2013). Revisión de análisis de tablas e introducción a modelos loglineales. Montevideo: Universidad de la Republica.

Busquets, J. M. (2009). El estado de bienestar y la seguridad social: Trayectorias, reformas y rereformas. En G. Bucheli y S. Harriet (Eds.), La seguridad social en el Uruguay. Constribuciones a su historia. Montevideo: Universidad de la Republica.

Ciganda, D. y Pardo, I. (2014). Emancipación y formación de hogares entre los jóvenes uruguayos: Las transformaciones recientes. En A. Pellegrino y C. Carela (Eds.), Hacerse adulto en Uruguay: Un estudio demográfico (pp. 15-38). Montevideo: UCUR.

Da Silveira, P. (2009). Padres, maestros y políticos. Montevideo: Aguilar.

Erikson, R., Goldthorpe, J. y Portocarero, H. (1979). Intergenerational class mobility in three western European societies: England, France and Sweden. British Journal of Sociology, 30(4), 415-441. https://doi.org/10.2307/589632

Esping-Andersen, G. (1990). The three worlds of welfare capitalism. Princeton, NJ: Princeton University Press.

Fernandez, T. y Boado, M. (2010). Trayectorias académicas y laborales de los jóvenes en Uruguay. Montevideo: AA Impresos.

Filardo, V. (2010). Transiciones a la adultez y educación. Montevideo: Trilce.

Filgueira, F., Pasturino, M., Opertti, R. y Vilaró, R. (2014). La educación prioridad de país: Aportes a la construcción de una Educación genuinamente inclusiva. Montevideo: Facultad de Ciencias Sociales, Universidad de la República.

Freire, P. (1987). Pedagogía del oprimido. Montevideo: Siglo XXI.

Gangl, M., Müller, W. y Raffe, D. (2003). Conclusions: explaining cross-national differences in school-to-work transitions. En W. Müller y M. Gangl (Eds.), Transitions from education to work in Europe: The integration of youth into eu labour markets (pp. 277-305). Oxford: Oxford University Press. https://doi.org/10.1093/0199252475.003.0010

Ganzeboom, H. (1996). Internationally comparable measures of occupational status for the 1988 international standard classification of occupations. Social Science Research, 25, 201-239. https://doi.org/10.1006/ssre.1996.0010

Goldthorpe, J. (1987). Social mobility and class structure in modern Britain. Oxford: Clarendon Press.

Goldthorpe, J. y Erikson, R. (1993). The constant flux. Nueva York, NY: Oxford University Press.

Kang, S. y Bishop, J. (1989). Vocational and academic education in high school: Complements or substitutes. Economics of Education Reviere, 8(2), 133-148. https://doi.org/10.1016/0272$7757(89) 90002-2$

Lin, N. (2001). Social capital. A theory of social structure and social action. Cambridge: Cambridge University Press. https://doi.org/10.1017/CBO9780511815447

Mancebo, E. y Goyeneche, G. (abril, 2010). Las políticas de inclusión educativa: entre la exclusión social y la innovación pedagógica. Comunicación presentada en IX Jornadas de la Facultad de Ciencias Sociales de la Universidad de la República. Montevideo. 
Menese, P. (2014). La acreditación de la educación media superior. Páginas de Educación, 6(2), 11 32.

Menese, P. y Ríos, Á. (2013). Evolución de la educación secundaria en los últimos 50 años. Desafíos para la próxima década. Montevideo: INEED

Midaglia, C. (2009). Entre la tradición, la modernización ingenua y los intentos de refundar la casa: la reforma social en el Uruguay de las últimas tres décadas. En C. Barba Solano (Ed.), Retos para la integración social de los pobres en América Latina. Buenos Aires: CLACSO.

Ríos, Á. (2014). Perfiles de riesgo educativo y trayectorias de los jóvenes durante la educación media superior. Páginas de Educación, 6(2), 33-54.

UNESCO. (2016). World social science report 2016: Challenging inequalities, pathways to a just world. Paris: UNESCO

Wright, E. O. (1997). Class counts: Comparative studies in class analysis. Cambridge: Cambridge University Press.

\section{Breve CV del autor}

\section{Pablo Menese Camargo}

Licenciado en Sociología por la Facultad de Ciencias Sociales de la Universidad de la República y candidato a Magister en Sociología con énfasis en Desigualdad y Movilidad Social por la Facultad de Ciencias Sociales de la Universidad de la República. Ejerce como Profesor Asistente en régimen de Dedicación Total en el Centro de Estudios de Políticas Educativas de la Universidad de la República. Su labor se centra fundamentalmente en desigualdades en las trayectorias educativas, y las tendencias contemporáneas de las desigualdades educativas. Entre sus publicaciones recientes se encuentra "Políticas sociales y su incidencia en la pobreza en Uruguay. Periodo 20062016" en coautoría con Pablo Ezquerra, y "Tire pá lante que empujan atrás. Tendencias en la desigualdad de oportunidades educativas en Uruguay" en coautoría con Santiago Cardozo. ORCID ID: 0000-0001-5940-8703. Email: pablo.menese@cut.edu.uy 